\title{
Resilience and Its Predictors in Caregivers of Patients with Advanced Oral Cavity Cancer during Survival
}

\author{
Li-Yun Lee ${ }^{1}$, Shu-Ching Chen ${ }^{2}$ \\ 1 Department of Nursing, China Medical University Beigang Hospital, Yunlin, Taiwan \\ 2 Department of Nursing, Chang Gung University of Science and Technology, Taoyuan, Taiwan
}

\section{Introduction:}

Anti-cancer treatments lead to varying levels of physical and psychological disturbance during treatment and prolong to survival period.

\section{Objectives:}

The purpose of the study was to identify resilience and related factors in primary caregivers of patients with advanced oral cavity cancer during the survival period.

\section{Methods:}

1. This study was a cross-sectional correlational design. Study was conducted to recruited subjects from RT outpatients department and cancer center in a medical center in northern Taiwan.

2. Eligible subjects were assessed for the Instrument Activities of Daily Living Scale (IADL), and background information form. Primary caregivers were assessed the related variables using the Resilience Scale(RS), Caregiver Reaction Assessment (CRA), Distress Thermometer(DT), Medical Outcomes Study

Social Support Survey(MOS SS), and a background information form.

\section{Results:}

1. Patients had moderate level of resilience was $36.5 \%$, with the lowest resilience was $57.7 \%$.

2. in "I usually take things in stride", "I feel that I can handle many things at a time", and "I am determined".

3. Caregivers had higher level of self-esteem burden $(\beta=0.397$, $p<0.001$ ), lower level of lack of family support burden $(\beta=0.378, p<0.001)$, and greater social support $(\beta=0.408$, $p<0.001$ ) were associated with positive resilience.

Table 4. Significant factors related to overall and five domains of resilience $(\mathrm{N}=104)$

\begin{tabular}{lrrrr}
\hline Variable & coefficient & $\begin{array}{l}95 \% \text { CI for } \\
\text { coefficient }\end{array}$ & $p$ & Adjusted $\mathrm{R}^{2}$ \\
\hline Overall resilience (RS) & & & & 0.418 \\
Caregivers' positive social interaction social support & 0.216 & $0.106-0.326$ & 0.001 & \\
Caregivers' lack of family support burden & -9.023 & $-13.265-4.782$ & 0.001 & \\
Caregivers' rotation with other family members (no vs. yes) & -5.482 & $-10.123--0.841$ & 0.001 & \\
Caregivers' self-esteem burden & 6.052 & $0.216-11.889$ & 0.021 & \\
Constant & 102.061 & $72.045-132.077$ & 0.042 &
\end{tabular}

Input independent variable: Patients' functional status (numbered scores), caregivers' rotation with other family members (no vs. yes), caregivers' caregiving burden (numbered scores), caregivers' social support (numbered scores), and caregivers' distress (numbered scores).
Conclusions: Caregiving burden and social support were the important factors associated with resilience of caregivers of oral cavity cancer patients. Survivorship care plan are recommended to help cope with caregiving burden.


Table 3. Distribution of Mean in Caregivers' Caregiving Burden, Social Support, Distress, and

\begin{tabular}{|c|c|c|}
\hline Variable & Mean/N & SD $/ \%$ \\
\hline Caregivers' Overall Caregiving Burden(CRA $)^{a}$ & 3.13 & 0.31 \\
\hline \multicolumn{3}{|l|}{ Different Domains of Care Burden } \\
\hline -Disrupted Schedule Burden & 2.92 & 0.67 \\
\hline -Financial Problem Burden & 2.76 & 0.78 \\
\hline -Lack of Family Support Burden & 2.29 & 0.59 \\
\hline -Health Problem Burden & 2.81 & 0.39 \\
\hline -Self-esteem Burden & 4.06 & 0.35 \\
\hline Overall Social Support(MOS-SS) ${ }^{b}$ & 66.17 & 15.94 \\
\hline \multicolumn{3}{|l|}{ Different Domains of Social Support } \\
\hline -Emotional/informational support & 63.94 & 20.35 \\
\hline -Tangible support & 57.93 & 22.75 \\
\hline -Affectionate support & 74.04 & 16.39 \\
\hline -Positive social interaction & 72.96 & 18.42 \\
\hline Distress $(\mathrm{DT})^{\mathrm{C}}$ & 3.06 & 2.76 \\
\hline Overall Resilience (RS) ${ }^{\mathrm{d}}$ & 118.60 & 12.07 \\
\hline \multicolumn{3}{|l|}{ Different Domains of Resilience } \\
\hline - Equanimity & 28.94 & 3.68 \\
\hline - Perseverance & 37.00 & 3.94 \\
\hline -Self-reliance & 28.42 & 3.58 \\
\hline - Meaningfulness & 14.54 & 2.21 \\
\hline - Existential aloneness & 9.69 & 1.48 \\
\hline \multicolumn{3}{|l|}{ Resilience Cut-offs } \\
\hline - Low resilience & 60 & 57.7 \\
\hline - Moderately low to moderate resilience & 38 & 36.5 \\
\hline - moderately high to high resilience & 6 & 5.8 \\
\hline
\end{tabular}
Support Survey. ${ }^{\mathrm{D} D T}$, Distress Thermometer, ${ }^{\mathrm{d}} \mathrm{RS}$, Resilience Scale 